\title{
Homicidio juvenil en México y su impacto en la esperanza de vida masculina: variaciones geográficas y factores asociados
}

\author{
Youth homicide in Mexico and its impact in male life \\ expectancy: geographical variations and associated \\ factors
}

Guillermo Julián González-Pérez', María Guadalupe Vega-López²

${ }^{1}$ Doctor en Ciencias de la Salud. Profesor-Investigador Titular, Centro Universitario de Ciencias de la Salud, Universidad de Guadalajara, México. $\bowtie$ iD

${ }^{2}$ Doctora en Ciencias de la Salud. ProfesoraInvestigadora Titular, Centro Universitario de Ciencias de la Salud, Universidad de Guadalajara, México. $\bowtie$ iD
RESUMEN Se analiza el impacto del homicidio juvenil (entre 15 y 29 años) en la esperanza de vida masculina en México y sus 32 estados en los trienios 2004-2006 y 2014-2016, y se identifican los factores que mejor explican las variaciones en los años de esperanza de vida masculina perdidos (AEVMP) por homicidio juvenil a nivel estatal en 2014-2016. A partir de datos oficiales, se calcularon la esperanza de vida temporaria masculina (EVTM) entre 0 y 85 años y los años de esperanza de vida masculina perdidos, total y por homicidio juvenil. Mediante un análisis de regresión lineal, se identificaron factores asociados con las variaciones de los años de esperanza de vida masculina perdidos por homicidio juvenil entre estados. La esperanza de vida temporaria masculina decreció entre ambos trienios en el país y en 25 estados, mientras que los años de esperanza de vida masculina perdidos por homicidio juvenil se incrementaron en 31 estados, en ocho de ellos la cifra fue superior a 0,5 en 2014-2016. Factores como la presencia del narcotráfico, de armas de fuego ilegales y la percepción de inseguridad explican las variaciones de los años de esperanza de vida masculina perdidos por homicidio juvenil entre estados en 2014-2016.

PALABRAS CLAVES Homicidio; Adolescente; Adulto Joven; Esperanza de Vida; Violencia; México.

\begin{abstract}
This study analyzes the impact of homicide in youth (between 15 and 29 years of age) on male life expectancy in Mexico and its 32 states in the periods 2004-2006 and 2014-2016, and identifies the factors that best explain the variations in the years of male life expectancy lost (YMLEL) due to youth homicide at the state level in 2014-2016. Based on official data, male temporary life expectancy (MTLE) between 0 to 85 years of age and years of male life expectancy lost, in total and due to youth homicide, were calculated in each three-year period. Through a linear regression analysis, factors associated with the variations of the years of male life expectancy lost due to youth homicide among states were identified. The male temporary life expectancy decreased between the three-year periods in the country and in 25 states; years of male life expectancy lost due to youth homicide increased in 31 states, in eight of them with a figure higher than 0.5 years in 2014-2016 period. Factors such as the presence of drug trafficking, illegal firearms and the perception of insecurity explain the variations in the years of male life expectancy lost due to youth homicide within the Mexican states in the 2014-2016 period.
\end{abstract}

KEY WORDS Homicide; Adolescent; Young Adult; Life Expectancy; Violence; Mexico. 


\section{INTRODUCCIÓN}

La violencia es, en la actualidad, una de las mayores preocupaciones de los ciudadanos de América Latina. El deterioro de la seguridad ha llevado al Programa de las Naciones Unidas para el Desarrollo (PNUD) a señalar que "el flanco débil de la región es la violencia, el crimen y la inseguridad"(1), una expresión lógica dada las altas tasas de homicidio que ha presentado la región en años recientes. En este contexto, México no es la excepción, pues la violencia en el país ha alcanzado niveles lo suficientemente elevados -en magnitud, intensidad y crueldad-como para convertir la inseguridad en un tema central del debate público a escala nacional ${ }^{(2)}$.

Los jóvenes mexicanos -en especial los hombres- son protagonistas en esta escalada de la criminalidad, pues existen condiciones en el país que aumentan el riesgo de que puedan ser víctimas de hechos delictivos o de verse implicados ellos mismos en la comisión de delitos: condiciones tanto de carácter estructural -por ejemplo, la persistente disparidad social ${ }^{(3)}-$, como de su entorno -en el ámbito familiar (violencia doméstica), en la escuela (acoso, intimidación) o en la comunidad (pandillerismo, narcomenudeo, características del barrio en que reside) - que hacen a los jóvenes extremadamente vulnerables a la violencia ${ }^{(4)}$. Varios estudios han documentado el incremento de la violencia homicida en la población juvenil mexicana en el presente siglo, lo que ha provocado que los homicidios sean la principal causa de muerte entre los hombres jóvenes mexicanos en los últimos años ${ }^{(4,5,6)}$.

Ciertamente, el homicidio es un caso extremo de violencia interpersonal, que pone de relieve los problemas que existen tanto en los distintos sectores sociales como en las relaciones específicas del ámbito privado $^{(7,8)}$. Cuando los homicidios o los intentos de homicidio involucran a los jóvenes, sus consecuencias sociales son mayores, pues la temprana edad de las víctimas contribuye en mayor grado al incremento de la "carga global" de muerte prematura, lesiones y discapacidad que padece la sociedad en su conjunto $^{(9)} \mathrm{y}$, en particular, a la pérdida de años de esperanza de vida, siendo este último uno de los indicadores más utilizados para medir el estado de salud y bienestar de una población.

A diferencia de la esperanza de vida femenina, la esperanza de vida masculina en México prácticamente se ha estancado en fechas recientes a nivel nacional, e incluso ha disminuido en algunas entidades federativas; aunque algunos estudios han documentado el peso de las muertes violentas en las edades jóvenes, en ninguno de ellos se ha procedido a evaluar la repercusión del homicidio juvenil en los cambios experimentados por la esperanza de vida masculina en la última década y las diferencias existentes a nivel estatal ${ }^{(10,11,12,13)}$.

A partir de la construcción del indicador años de esperanza de vida masculina perdidos (AEVMP) a causa del homicidio juvenil, el presente trabajo tiene como objetivos analizar el impacto del homicidio de jóvenes, entre los 15 y 29 años, en la esperanza de vida masculina a nivel nacional y por entidad federativa en los trienios 2004-2006 y 2014-2016, e identificar los factores socioeconómicos que mejor explican las variaciones encontradas en dicho indicador a nivel estatal en el trienio 2014-2016.

\section{MATERIAL Y MÉTODOS}

Este es un estudio observacional y descriptivo, basado en fuentes secundarias de información, aunque también puede ser considerado -en lo concerniente al análisis a nivel estatal- como un estudio de tipo ecológico. Tanto la información sobre mortalidad como los datos de población para los años estudiados se obtuvieron esencialmente de las bases de datos oficiales (Cubos Dinámicos) de la Dirección General de Información en Salud de la Secretaría de Salud, cuyo referente, en el caso de la población, son las estimaciones y proyecciones del Consejo Nacional de Población. La única excepción 
fueron las defunciones de 2016, que se obtuvieron de la base de datos del Instituto Nacional de Geografía y Estadística, dado que aún no se encontraba disponible la información en la Dirección General de Información en Salud al momento del estudio ${ }^{(14,15,16)}$; cabe señalar que esto no genera ningún problema de comparabilidad con la fuente utilizada para los años previos puesto que, en todos los casos, las cifras oficiales son cotejadas por ambas instituciones $y$, por tanto, son las mismas.

En particular, los homicidios se clasificaron de acuerdo con la Clasificación Internacional de Enfermedades 10a Revisión (CIE-10): X85-Y09, Y87.1 ${ }^{(17)}$. Asimismo, vale señalar que en este estudio los términos "entidad federativa" y "estado" se usan indistintamente cuando se hace referencia a la división político-administrativa del país.

De acuerdo con los criterios de la Organización Panamericana de la Salud (OPS), los registros de mortalidad que tienen una cobertura de defunciones superior al $90 \%$ o una proporción de causas de muerte mal definidas menor al $10 \%$ pueden considerarse como de alta calidad ${ }^{(18)}$; en tal sentido, la información sobre mortalidad en México en las últimas décadas puede considerarse adecuada, por lo que se decidió utilizar en esta investigación los datos oficiales de mortalidad reportados. Ante la existencia de defunciones de edad no especificada (un porcentaje menor al $1 \%$ del total de defunciones en cada trienio, y en el caso de los homicidios, del $5 \%$ en el trienio 2014-2016 y del $2 \%$ en el trienio 2004-2006) (14), se corrigió dicha situación redistribuyendo proporcionalmente las defunciones de edad ignorada, en general y por homicidio, según el peso relativo de cada grupo etario. Se trata de un procedimiento empleado comúnmente en estudios de mortalidad, que no cambia la tendencia de los datos y se basa en los principios de expansión del muestreo aleatorio simple $\mathrm{e}^{(19)}$.

Dado el hecho de que el concepto de juventud puede tener diferentes definiciones y límites en cuanto a la edad, se consideró en este estudio como jóvenes a la población entre 15 y 29 años, en concordancia con los criterios empleados por la Unión
Europea $^{(4)}$, organismos internacionales como la Comisión Económica para América Latina (CEPAL), y la Organización Iberoamericana de la Juventud ${ }^{(20)} y$, en particular, el Instituto Nacional de Estadística y Geografía (INEGI) de México ${ }^{(21)}$, lo cual permite mantener una comparabilidad con diversos proyectos estadísticos a nivel internacional para la población de estudio.

Si bien en principio se calcularon tasas de homicidio masculino total y para los grupos de edad 15 a 19, 20 a 24 y 25 a 29 años, y para los trienios 1989-1991, 1994-1996, 1999-2001, 2004-2006, 2009-2011 у 20142016, el estudio se centró sustancialmente en los trienios 2004-2006 y 2014-2016, debido a la posibilidad de comparar la situación más reciente del país con la existente diez años atrás, cuando las tasas de homicidio juvenil alcanzaron su nivel más bajo, además de poder trabajar con datos referidos a una sola revisión de la Clasificación Internacional de Enfermedades (CIE-10)

Con los datos existentes sobre defunciones y población, se construyeron tablas de mortalidad masculina abreviadas para México y sus 32 entidades federativas en los trienios señalados, empleando para ello el programa EPIDAT v3.1 ${ }^{(22)}$. La construcción de tablas de mortalidad trienales tuvo el objeto de reducir posibles variaciones aleatorias de la mortalidad que pudieran distorsionar la tendencia de su comportamiento.

A partir de las tablas de mortalidad, y de acuerdo con el método propuesto por Arriaga, se calcularon tanto la esperanza de vida temporaria masculina (EVTM) entre 0 y 85 años como los años de esperanza de vida masculina perdidos (AEVMP) entre las dos edades (en general, por homicidios y por grupos de edad en cada trienio). El número de AEVMP se calculó multiplicando la proporción de personas que fallecen entre las edades $x$ y $x+n$ en la población estacionaria de la tabla de mortalidad, por la diferencia entre el número medio de años que podrían haber vivido desde $x$ en caso de no fallecer y el promedio de años realmente vividos entre $x y x+n$ por la población que muere en ese grupo de edad ${ }^{(23,24)}$. Ambos indicadores 
fueron calculados mediante el programa EPIDAT v3.1. El método de Arriaga ha sido empleado por diversos autores y aparece descrito en detalle en la literatura ${ }^{(10,12,13,23,24)}$.

En tal sentido, los AEVMP representan la diferencia entre el máximo posible de años que se pueden vivir entre dos edades (o sea, 85 años en este caso) y la EVTM, que es el promedio de años vividos por la población masculina entre esas edades ${ }^{(23)}$, es decir, los AEVMP son los años no vividos. La suma de estos años no vividos, por grupos de edad y causa, permitió obtener los AEVMP en total, así como los atribuibles a homicidios para cada entidad federativa $y$, en particular, los AEVMP que corresponderían al grupo de 15 a 29 años (en general y por homicidios). Los AEVMP por homicidio se convierten así en un indicador robusto del nivel del homicidio juvenil masculino en el trienio 2014-2016.

En este estudio se calcularon los AEVMP asumiendo una mortalidad nula entre $0 \mathrm{y}$ 85 años. Este supuesto facilita la interpretación del indicador: el total de AEVMP entre las edades " 0 " y " 85 " es igual a la diferencia entre el número máximo de años que se pueden vivir entre ambas edades si se elimina la mortalidad (en este caso 85 años) y la EVTM entre " 0 " y " 85 " (es decir, el promedio de años que realmente se viven) ${ }^{(25)}$.

Por otra parte, se realizó un análisis de regresión lineal múltiple (regresión de mínimos cuadrados ponderada), utilizando el método conocido como "pasos sucesivos" (o "stepwise") para identificar factores socioeconómicos que mejor pudieran explicar las variaciones de los AEVMP por homicidios en el grupo de 15 a 29 años entre las entidades federativas en el trienio 2014-2016. Este procedimiento, utilizado por diferentes autores en el campo de la salud ${ }^{(26,27)}$, toma la variable que presenta la mayor correlación con la variable dependiente y la incluye en el modelo predictivo, y va incorporando nuevas variables a la ecuación, en la medida en que estas puedan producir un cambio estadísticamente significativo en la regresión. La probabilidad de la prueba $F(0,05$ entrada; 0,10 eliminación) fue el criterio elegido para seleccionar las variables definitivamente incluidas en la ecuación.

Las variables seleccionadas para el análisis reflejan condiciones socioeconómicas de cada entidad federativa alrededor de las fechas en que está centrado el estudio: porcentaje de población de 18 años o más que se siente insegura en su entorno (2015) ${ }^{(28)}$; porcentaje de ineficiencia terminal en bachiIlerato (2016) ${ }^{(29)}$; porcentaje de jóvenes de 15 a 24 años que no estudian ni tienen un rol laboral permanente $(2016)^{(29)}$, porcentaje de población en condición de pobreza (2016) y el coeficiente de Gini como indicador de la desigualdad en los ingresos $(2016)^{(30)}$. Además, las variables "hectáreas cultivadas con marihuana y opiáceos destruidas por el ejército (por 100.000 habitantes)", "armas de fuego confiscadas por el ejército, por 100.000 habitantes"(29) e "índice de impunidad", las dos primeras calculadas para el bienio 20142015, y la última para 2012 (último dato disponible) $)^{(31)}$ fueron incluidas como "proxies" de la magnitud de las actividades relacionadas con el tráfico de drogas, la disponibilidad de armas de fuego y el funcionamiento del sistema judicial, respectivamente. En particular, el índice de impunidad fue construido al restarle a 1 el resultado de dividir el número de delincuentes sentenciados por homicidio entre el número de homicidios registrados en cada período estudiado y posteriormente multiplicarlo por 100 .

La regresión fue ponderada por la población de 15 a 29 años existente en cada estado en 2015. La prueba " $\mathrm{t}$ " de Student fue usada para determinar si los coeficientes de regresión calculados eran significativamente diferentes de cero y la prueba de Durbin-Watson, para evaluar la autocorrelación de las variables en el modelo. Igualmente, se evaluó la posible multicolinealidad mediante el índice de condición. Para el procesamiento de la información y el análisis estadístico de los datos, se utilizó el programa SPSS v23.

El protocolo de investigación "Violencia, juventud y armas de fuego en México", del que deriva este artículo, fue aprobado por el Centro de Estudios en Salud, Población y Desarrollo Humano de la Universidad de Guadalajara y registrado con la clave SyP-2017-004. 
El proyecto se ajusta a los lineamientos éticos expuestos en el Reglamento de la Ley General de Salud en materia de investigación para la salud de México, pues se consideró como "investigación sin riesgo", en tanto se analizan datos secundarios, empleándose técnicas y métodos de investigación documental-con datos protegidos- que no afectan a ningún individuo.

\section{RESULTADOS}

En la Figura 1 se puede observar que, entre 1990 y 2005, existe un claro comportamiento descendente de las tasas de homicidio masculinas, tanto en general como entre los 15 y 29 años; sin embargo, a partir del trienio 2004-2006, la tendencia de las tasas es al alza, y aunque las tasas más elevadas se haIlan en el trienio 2009-2011, las observadas en el trienio 2014-2016 -en general, y en los grupos de edades analizados- son superiores a las reportadas en los primeros años de la década de 1990.

En el grupo de 25 a 29 años se observan las tasas más elevadas en todo el período estudiado, alcanzando en los dos últimos trienios valores superiores a 65 homicidios por 100.000 habitantes, cifras que en la práctica son el doble de las tasas observadas en la población en general. En segundo lugar, se observan las tasas del grupo de 20 a 24 años, mientras las tasas de 15 a 19 años, aunque inferiores al promedio nacional, alcanzan en el trienio 2014-2016 su valor más alto en todo el lapso analizado.

Asimismo, las tasas del grupo de 15 a 29 años en su conjunto siempre se han mantenido claramente por encima de la tasa promedio nacional. En la práctica, todas las tasas del trienio 2014-2016 duplican las registradas en 2004-2006, siendo el grupo de 20 a 24 años

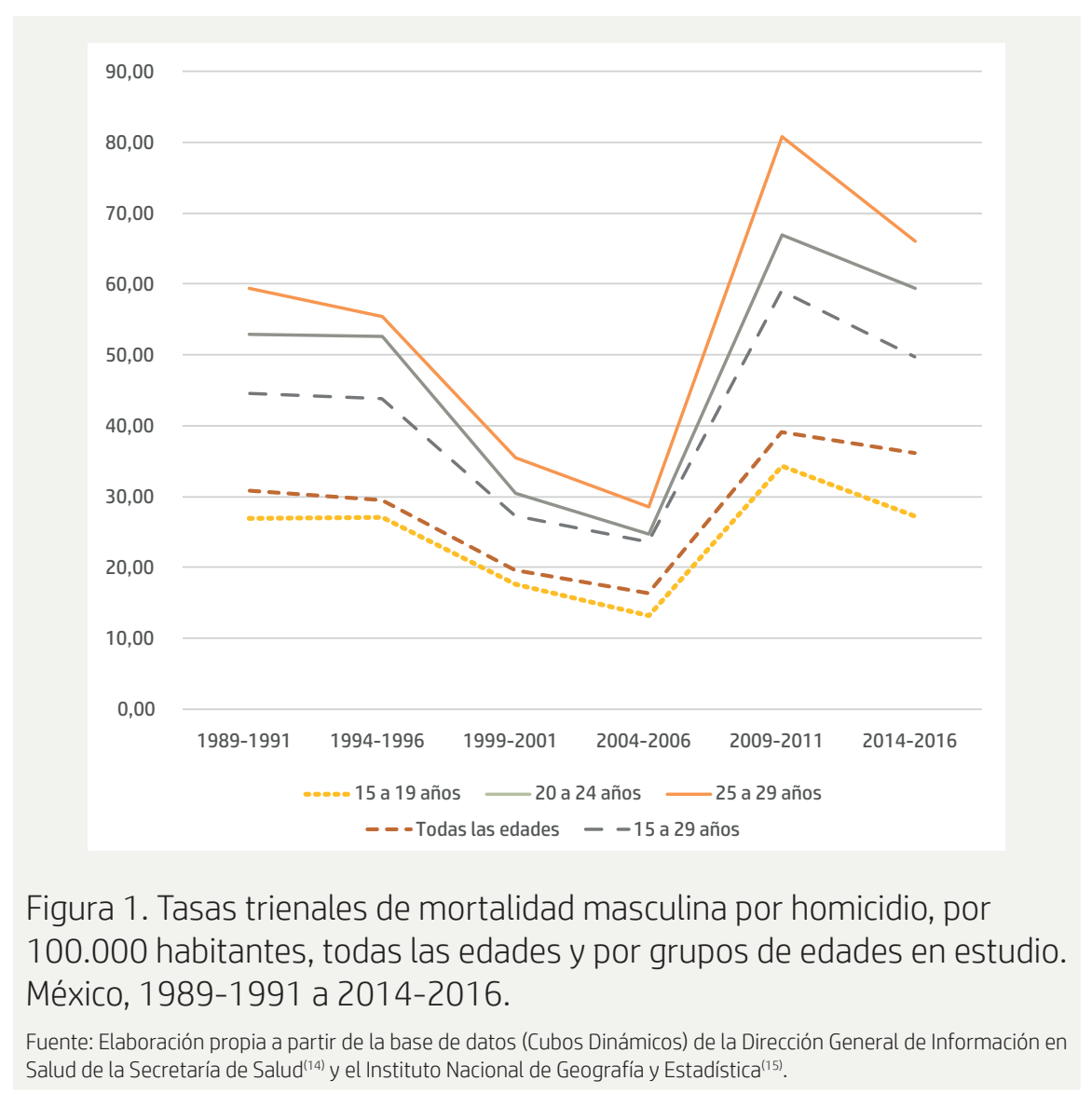


el que presenta el incremento más marcado.

En la Tabla 1 se puede apreciar que, tanto a nivel nacional como en 25 estados del país, la EVTM entre 0 y 85 años disminuyó entre los dos trienios estudiados. En nueve entidades federativas, la reducción de la EVTM fue superior a un año, y se destacan los casos de Guerrero y Colima que perdieron, entre 2004-2006 y 2014-2016, 3,7 y 2,4 años respectivamente. En cinco estados, la EVTM

Tabla 1. Esperanza de vida temporaria masculina (EVTM), años de esperanza de vida masculina perdidos (AEVMP) y años de esperanza de vida masculina perdidos por homicidio (AEVMPH) entre 0 y 85 años, a nivel nacional y por entidad federativa. México, 2004-2006 y 2014-2016.

\begin{tabular}{|c|c|c|c|c|c|c|c|}
\hline \multirow[t]{2}{*}{ Estados } & \multicolumn{2}{|c|}{ EVTM } & \multirow{2}{*}{$\frac{\text { Diferencia }}{\text { EVTM }}$} & \multicolumn{2}{|c|}{ AEVMP } & \multicolumn{2}{|c|}{ AEVMPH } \\
\hline & 2004-2006 & 2014-2016 & & 2004-2006 & 2014-2016 & 2004-2006 & 2014-2016 \\
\hline México & 71,51 & 71,16 & $-0,35$ & 13,49 & 13,84 & 0,55 & 1,03 \\
\hline Aguascalientes & 72,56 & 72,45 & $-0,12$ & 12,44 & 12,55 & 0,12 & 0,22 \\
\hline Baja California & 68,94 & 69,65 & 0,71 & 16,06 & 15,35 & 0,82 & 1,34 \\
\hline Baja California Sur & 71,10 & 71,92 & 0,82 & 13,90 & 13,08 & 0,30 & 1,17 \\
\hline Campeche & 72,88 & 71,78 & $-1,10$ & 12,12 & 13,22 & 0,28 & 0,46 \\
\hline Coahuila & 71,74 & 71,00 & $-0,74$ & 13,26 & 14,00 & 0,27 & 0,64 \\
\hline Colima & 72,22 & 69,85 & $-2,37$ & 12,78 & 15,15 & 0,45 & 2,45 \\
\hline Chiapas & 71,78 & 70,99 & $-0,80$ & 13,22 & 14,01 & 0,49 & 0,59 \\
\hline Chihuahua & 68,92 & 68,62 & $-0,30$ & 16,08 & 16,38 & 0,97 & 2,64 \\
\hline Ciudad de México & 71,13 & 70,27 & $-0,87$ & 13,87 & 14,73 & 0,47 & 0,72 \\
\hline Durango & 71,87 & 71,60 & $-0,27$ & 13,13 & 13,40 & 0,74 & 0,93 \\
\hline Guanajuato & 72,42 & 71,13 & $-1,29$ & 12,58 & 13,87 & 0,24 & 1,05 \\
\hline Guerrero & 73,65 & 69,92 & $-3,73$ & 11,35 & 15,08 & 1,35 & 3,91 \\
\hline Hidalgo & 72,38 & 72,70 & 0,31 & 12,62 & 12,30 & 0,19 & 0,41 \\
\hline Jalisco & 71,65 & 71,10 & $-0,55$ & 13,35 & 13,90 & 0,37 & 0,87 \\
\hline Estado de México & 70,90 & 72,24 & 1,34 & 14,10 & 12,76 & 0,76 & 0,93 \\
\hline Michoacán & 71,73 & 71,54 & $-0,18$ & 13,27 & 13,46 & 1,13 & 1,44 \\
\hline Morelos & 72,53 & 71,15 & $-1,38$ & 12,47 & 13,85 & 0,50 & 1,55 \\
\hline Nayarit & 72,52 & 72,80 & 0,28 & 12,48 & 12,20 & 0,74 & 0,72 \\
\hline Nuevo León & 72,44 & 72,36 & $-0,08$ & 12,56 & 12,64 & 0,16 & 0,60 \\
\hline Oaxaca & 71,63 & 71,45 & $-0,18$ & 13,37 & 13,55 & 0,95 & 1,16 \\
\hline Puebla & 70,25 & 70,61 & 0,36 & 14,75 & 14,39 & 0,38 & 0,60 \\
\hline Querétaro & 71,78 & 71,70 & $-0,09$ & 13,22 & 13,30 & 0,24 & 0,35 \\
\hline Quintana Roo & 72,45 & 71,87 & $-0,58$ & 12,55 & 13,13 & 0,40 & 0,49 \\
\hline San Luis Potosí & 73,06 & 72,77 & $-0,29$ & 11,94 & 12,23 & 0,37 & 0,61 \\
\hline Sinaloa & 73,06 & 71,45 & $-1,61$ & 11,94 & 13,55 & 0,90 & 2,42 \\
\hline Sonora & 70,47 & 70,35 & $-0,12$ & 14,53 & 14,65 & 0,57 & 1,15 \\
\hline Tabasco & 71,33 & 69,85 & $-1,48$ & 13,67 & 15,15 & 0,32 & 0,79 \\
\hline Tamaulipas & 71,71 & 71,17 & $-0,55$ & 13,29 & 13,83 & 0,56 & 1,18 \\
\hline Tlaxcala & 72,30 & 72,52 & 0,21 & 12,70 & 12,48 & 0,23 & 0,39 \\
\hline Veracruz & 71,27 & 70,44 & $-0,83$ & 13,73 & 14,56 & 0,28 & 0,74 \\
\hline Yucatán & 72,82 & 71,86 & $-0,96$ & 12,18 & 13,14 & 0,11 & 0,14 \\
\hline Zacatecas & 72,84 & 71,73 & $-1,11$ & 12,16 & 13,27 & 0,36 & 1,41 \\
\hline
\end{tabular}


fue inferior a 70 años, siendo Chihuahua el estado con la menor EVTM (68,6 años).

En concordancia con lo anterior, a nivel nacional y en 25 estados se incrementaron los AEVMP entre ambos trienios. En particular, Ios AEVMP por homicidio aumentaron tanto a nivel nacional como en 31 entidades federativas, en 16 de las cuales las cifras de 2014-2016 más que duplicaron las calculadas para 2004-2006. En 14 de los 25 estados que vieron reducida su EVTM, los AEVMP por homicidio fueron en 2014-2016 más del doble de los calculados para 2004-2006. Sobresale el estado de Colima, donde el número de AEVMP por homicidio se quintuplicó en 2014-2016 con respecto a 2004-2006.

Aunque entre los 15 y 29 años los AEVMP se incrementaron a escala nacional y en la gran mayoría de las entidades federativas (excepto en cuatro), el aumento de los AEVMP por homicidios en este grupo de edad fue mucho más acentuado: solo en un estado (Nayarit) las cifras del 2014-2016 no rebasaron las calculadas para 2004-2006. En 16 estados, el incremento de los AEVMP por homicidios entre 15 y 29 años entre ambos trienios al menos dobla el observado para los AEVMP por el grupo de 15 a 29 en general (Tabla 2).

Un reflejo de ello es que mientras a nivel nacional el aumento de los AEVMP por el grupo de 15 a 29 años entre ambos trienios fue del 18\%, los AEVMP por homicidios en este mismo grupo de edad se duplicaron en dicho lapso. Resaltan los casos de Guerrero, Chihuahua y Sinaloa, con más de un AEVMP por homicidio juvenil en 2014-2016, y Colima, en donde el número de AEVMP por homicidios en este grupo de edad fue en 2014-2016 casi 11 veces mayor al valor de 2004-2006. En tal sentido, cabe destacar que en ocho estados la cifra de AEVMP por homicidio juvenil rebasó el medio año $(0,5)$, un valor no alcanzado por ninguna entidad federativa en el trienio 2004-2006

A nivel nacional, esto significa que los homicidios masculinos en el grupo de 15 a 29 años fueron responsables -en el trienio 2014-2016- del 3\% del total de AEVMP en el país, del $40 \%$ del total de AEVMP por homicidios y de algo más de la cuarta parte del total de AEVMP en el grupo de 15 a 29 años, cifras estas todas superiores a las calculadas para el trienio 2004-2006 (Tabla 3).

Con la excepción de Nayarit, en el resto de las entidades federativas los AEVMP por homicidios en el grupo de 15 a 29 años incrementaron en 2014-2016 su peso proporcional en el total de AEVMP con respecto a 2004-2006, sobresaliendo los casos de Guerrero (en donde más del 10\% del total de AEVMP en el último trienio fueron causados por los homicidios masculinos entre 15 y 29 años), Sinaloa y Chihuahua (más del $7 \%$ ) y Colima (más del $6 \%$ ).

Asimismo, en 14 entidades, los AEVMP por homicidios entre 15 y 29 años en 20142016 representaron más del $40 \%$ del total de AEVMP por homicidios (por 6 entidades en 2004-2006). Por otra parte, en todos los estados se observó un incremento entre ambos trienios estudiados del peso relativo de los AEVMP por homicidios en el grupo de 15 a 29 años con relación al total de AEVMP por dicho grupo etario: en Guerrero, por ejemplo, en 2014-2016 representaron cerca de las dos terceras partes de todos los AEVMP en el grupo de 15 a 29 años (comparado con $29 \%$ en 2004-2006), en Sinaloa y Chihuahua prácticamente la mitad $(23 \%$ y $26 \%$ respectivamente en 2004-2006) y en Colima el $45 \%$ (7\% en 2004-2006).

No obstante, debe señalarse que existen estados en los cuales, en ambos trienios, el número de AEVMP por homicidios en el grupo de 15 a 29 años fue relativamente bajo y por ende su peso proporcional, tanto en los AEVMP totales como en los del grupo 15 a 29 es reducido: fueron los casos de Yucatán, Aguascalientes y Tlaxcala, en los que las cifras de AEVMP por homicidio en el grupo 15 a 29 han sido iguales o menores a 0,10 en ambos trienios $y$, por tanto, han representado menos del $1 \%$ del total de AEVMP, y menos del $10 \%$ del total de AEVMP entre 15 y 29 años en cada uno de ellos.

Tras el análisis de regresión lineal múltiple (Tabla 4), se obtuvo un modelo que revela aquellos factores sociales - de los analizados en este estudio- que mejor explican la variabilidad por entidad federativa del número de 
Tabla 2. Años de esperanza de vida masculina perdidos (AEVMP) de 15 a 29 años, y años de esperanza de vida masculina perdidos por homicidio (AEVMPH) de 15 a 29 años a nivel nacional y por entidad federativa. México, 2004-2006 y 2014-2016.

\begin{tabular}{|c|c|c|c|c|c|c|}
\hline Estados & $\begin{array}{c}\text { AEVMP } \\
2004-2006\end{array}$ & $\begin{array}{c}\text { AEVMP } \\
2014-2016\end{array}$ & $\begin{array}{c}\text { Razón } \\
\text { 2014-2016/ } \\
\text { 2004-2006 }\end{array}$ & $\begin{array}{c}\text { AEVMPH } \\
2004-2006\end{array}$ & $\begin{array}{c}\text { AEVMPH } \\
2014-2016\end{array}$ & $\begin{array}{c}\text { Razón } \\
\text { 2014-2016/ } \\
\text { 2004-2006 }\end{array}$ \\
\hline México & 1,28 & 1,52 & 1,18 & 0,20 & 0,41 & 2,10 \\
\hline Aguascalientes & 1,06 & 1,34 & 1,27 & 0,03 & 0,10 & 3,98 \\
\hline Baja California & 1,52 & 1,50 & 0,99 & 0,32 & 0,46 & 1,46 \\
\hline Baja California Sur & 1,16 & 1,47 & 1,27 & 0,10 & 0,49 & 4,69 \\
\hline Campeche & 1,18 & 1,32 & 1,12 & 0,08 & 0,13 & 1,61 \\
\hline Coahuila & 0,98 & 1,26 & 1,28 & 0,11 & 0,29 & 2,56 \\
\hline Colima & 1,14 & 2,08 & 1,82 & 0,08 & 0,94 & 11,81 \\
\hline Chiapas & 1,62 & 1,42 & 0,88 & 0,18 & 0,19 & 1,04 \\
\hline Chihuahua & 1,85 & 2,33 & 1,26 & 0,42 & 1,16 & 2,76 \\
\hline Ciudad de México & 1,07 & 1,40 & 1,31 & 0,22 & 0,31 & 1,44 \\
\hline Durango & 1,23 & 1,43 & 1,16 & 0,28 & 0,37 & 1,32 \\
\hline Guanajuato & 1,14 & 1,72 & 1,52 & 0,10 & 0,43 & 4,17 \\
\hline Guerrero & 1,32 & 2,49 & 1,89 & 0,39 & 1,57 & 4,08 \\
\hline Hidalgo & 1,13 & 1,14 & 1,01 & 0,06 & 0,13 & 2,30 \\
\hline Jalisco & 1,28 & 1,55 & 1,21 & 0,13 & 0,35 & 2,70 \\
\hline Estado de México & 1,24 & 1,30 & 1,05 & 0,28 & 0,38 & 1,36 \\
\hline Michoacán & 1,66 & 1,76 & 1,06 & 0,36 & 0,54 & 1,50 \\
\hline Morelos & 1,21 & 1,63 & 1,35 & 0,16 & 0,55 & 3,44 \\
\hline Nayarit & 1,49 & 1,29 & 0,87 & 0,26 & 0,23 & 0,90 \\
\hline Nuevo León & 0,85 & 1,10 & 1,29 & 0,05 & 0,27 & 5,05 \\
\hline Oaxaca & 1,47 & 1,43 & 0,97 & 0,27 & 0,34 & 1,25 \\
\hline Puebla & 1,19 & 1,31 & 1,10 & 0,11 & 0,20 & 1,78 \\
\hline Querétaro & 1,24 & 1,25 & 1,00 & 0,09 & 0,14 & 1,53 \\
\hline Quintana Roo & 1,23 & 1,27 & 1,03 & 0,15 & 0,19 & 1,24 \\
\hline San Luis Potosí & 1,22 & 1,30 & 1,06 & 0,14 & 0,27 & 1,94 \\
\hline Sinaloa & 1,22 & 2,13 & 1,74 & 0,32 & 1,08 & 3,33 \\
\hline Sonora & 1,28 & 1,49 & 1,17 & 0,23 & 0,41 & 1,78 \\
\hline Tabasco & 1,64 & 1,88 & 1,15 & 0,11 & 0,30 & 2,76 \\
\hline Tamaulipas & 1,24 & 1,56 & 1,26 & 0,24 & 0,56 & 2,39 \\
\hline Tlaxcala & 1,10 & 1,16 & 1,05 & 0,07 & 0,10 & 1,57 \\
\hline Veracruz & 1,28 & 1,64 & 1,27 & 0,08 & 0,31 & 3,66 \\
\hline Yucatán & 0,96 & 1,01 & 1,05 & 0,04 & 0,05 & 1,20 \\
\hline Zacatecas & 1,36 & 1,93 & 1,41 & 0,11 & 0,68 & 5,96 \\
\hline
\end{tabular}

AEVMP por homicidio en el grupo de 15 a 29 años en el trienio 2014-2016. De acuerdo con el coeficiente de regresión estandarizado los resultados indican que la variable "hectáreas cultivadas de marihuana y opiáceos destruidas por el ejército" es el factor de mayor peso para explicar las variaciones interestatales de los AEVMP por homicidios en este trienio. Asimismo, las variables "porcentaje de población de 18 años o más que se siente 
Tabla 3. Porcentaje que representan los años de esperanza de vida masculina perdidos de 15 a 29 años por homicidio (AEVMPH 15 a 29) del total de años de esperanza de vida masculina perdidos (AEVMP), del total de años de esperanza de vida masculina perdidos por homicidio (AEVMPH) y del total de años de esperanza de vida masculina perdidos de 15 a 29 (AEVMP 15 a 29), a nivel nacional y por entidad federativa. México, 2004-2006 y 2014-2016.

\begin{tabular}{|c|c|c|c|c|c|c|}
\hline Estados & $\begin{array}{c}\text { AEVMP } \\
2004-2006 \\
(\%)\end{array}$ & $\begin{array}{c}\text { AEVMP } \\
2014-2016 \\
(\%)\end{array}$ & $\begin{array}{c}\text { AEVMPH } \\
2004-2006 \\
(\%)\end{array}$ & $\begin{array}{c}\text { AEVMPH } \\
2014-2016 \\
(\%)\end{array}$ & $\begin{array}{c}\text { AEVMPH } \\
15 \text { a } 29 \\
2004-2006 \\
(\%)\end{array}$ & $\begin{array}{c}\text { AEVMPH } \\
15 \text { a } 29 \\
2014-2016 \\
(\%)\end{array}$ \\
\hline México & 1,46 & 2,99 & 35,98 & 40,03 & 15,32 & 27,30 \\
\hline Aguascalientes & 0,21 & 0,82 & 21,03 & 46,49 & 2,43 & 7,64 \\
\hline Baja California & 1,97 & 3,01 & 38,63 & 34,50 & 20,82 & 30,70 \\
\hline Baja California Sur & 0,74 & 3,71 & 34,10 & 41,33 & 8,96 & 33,00 \\
\hline Campeche & 0,67 & 0,99 & 28,75 & 28,45 & 6,88 & 9,96 \\
\hline Coahuila & 0,86 & 2,09 & 42,73 & 46,03 & 11,65 & 23,22 \\
\hline Colima & 0,63 & 6,24 & 17,88 & 38,52 & 7,00 & 45,44 \\
\hline Chiapas & 1,34 & 1,31 & 35,81 & 30,93 & 10,95 & 12,95 \\
\hline Chihuahua & 2,62 & 7,10 & 43,30 & 44,10 & 22,75 & 49,86 \\
\hline Ciudad de México & 1,56 & 2,11 & 45,74 & 43,46 & 20,19 & 22,17 \\
\hline Durango & 2,12 & 2,75 & 37,66 & 39,63 & 22,59 & 25,79 \\
\hline Guanajuato & 0,81 & 3,07 & 42,21 & 40,50 & 8,95 & 24,66 \\
\hline Guerrero & 3,40 & 10,44 & 28,51 & 40,24 & 29,31 & 63,30 \\
\hline Hidalgo & 0,45 & 1,07 & 30,16 & 31,75 & 5,04 & 11,47 \\
\hline Jalisco & 0,96 & 2,49 & 34,31 & 39,81 & 10,04 & 22,40 \\
\hline Estado de México & 1,98 & 2,97 & 36,83 & 40,71 & 22,44 & 29,01 \\
\hline Michoacán & 2,70 & 4,00 & 31,68 & 37,41 & 21,52 & 30,53 \\
\hline Morelos & 1,29 & 4,00 & 32,36 & 35,80 & 13,29 & 33,99 \\
\hline Nayarit & 2,05 & 1,90 & 34,76 & 32,25 & 17,12 & 17,86 \\
\hline Nuevo León & 0,42 & 2,13 & 32,98 & 45,19 & 6,25 & 24,49 \\
\hline Oaxaca & 2,06 & 2,53 & 28,90 & 29,46 & 18,72 & 24,01 \\
\hline Puebla & 0,77 & 1,40 & 29,62 & 33,32 & 9,48 & 15,39 \\
\hline Querétaro & 0,67 & 1,02 & 37,31 & 38,31 & 7,12 & 10,87 \\
\hline Quintana Roo & 1,19 & 1,41 & 37,32 & 37,45 & 12,13 & 14,54 \\
\hline San Luis Potosí & 1,16 & 2,20 & 37,63 & 44,10 & 11,35 & 20,67 \\
\hline Sinaloa & 2,71 & 7,95 & 36,08 & 44,55 & 26,46 & 50,64 \\
\hline Sonora & 1,61 & 2,83 & 41,03 & 36,03 & 18,22 & 27,77 \\
\hline Tabasco & 0,78 & 1,95 & 33,34 & 37,71 & 6,54 & 15,73 \\
\hline Tamaulipas & 1,77 & 4,07 & 42,36 & 47,58 & 18,95 & 36,09 \\
\hline Tlaxcala & 0,52 & 0,83 & 28,89 & 26,90 & 6,02 & 8,97 \\
\hline Veracruz & 0,61 & 2,09 & 29,30 & 41,20 & 6,49 & 18,64 \\
\hline Yucatán & 0,32 & 0,36 & 34,24 & 32,50 & 4,08 & 4,67 \\
\hline Zacatecas & 0,93 & 5,11 & 31,58 & 47,94 & 8,33 & 35,14 \\
\hline
\end{tabular}

insegura en su entorno" y "armas de fuego confiscadas por el ejército" también entraron en el modelo, el cual -de acuerdo con el coeficiente de determinación ajustado ( $\mathrm{R}^{2}$ ajustado)- explica el $73 \%$ de las variaciones de la variable dependiente (AEVMP por homicidios en el grupo de 15 a 29 años).

Por el contrario, el resto de las variables analizadas no tienen un papel relevante para explicar las diferencias observadas en los 


\begin{tabular}{|c|c|c|c|}
\hline Variables socioeconómicas & $\begin{array}{l}\text { Coeficiente } \\
\text { de regresión } \\
\text { estandarizado }\end{array}$ & $\mathrm{t}$ & Valor de $p$ \\
\hline \multicolumn{4}{|l|}{ Variables en la ecuación } \\
\hline Armas de fuego confiscadas (por 100.000 habitantes) & 0,193 & 2,04 & 0,046 \\
\hline $\begin{array}{l}\text { Hectáreas destruidas de mariguana y opiáceos (por } \\
100.000 \text { habitantes) }\end{array}$ & 0,751 & 7,90 & 0,000 \\
\hline Percepción de inseguridad (\%) & 0,223 & 2,33 & 0,027 \\
\hline \multicolumn{4}{|l|}{ Variables excluidas de la ecuación } \\
\hline $\begin{array}{l}\text { Jóvenes de } 15 \text { a } 24 \text { años que no estudian ni tienen un } \\
\text { rol laboral permanente (\%) }\end{array}$ & 0,101 & 1,06 & 0,297 \\
\hline Índice de impunidad (\%) & 0,040 & 0,38 & 0,706 \\
\hline Población en condición de pobreza (\%) & $-0,018$ & $-0,19$ & 0,852 \\
\hline Coeficiente de Gini & 0,055 & 0,57 & 0,571 \\
\hline Ineficiencia terminal en bachillerato (\%) & 0,062 & 0,65 & 0,520 \\
\hline \multicolumn{4}{|c|}{$\begin{array}{l}\text { Fuente: Elaboración propia a partir de la base de datos (Cubos Dinámicos) de la Dirección General de Información en } \\
\text { Salud de la Secretaria de Salud(14) y el Instituto Nacional de Geografía y Estadistica }{ }^{(15)} \text {. } \\
\text { Análisis de varianza: grados de libertad=3; prueba } F=29,20 ; p=0,000 \text {. } \\
\text { Durbin-Watson test: } 2,04 \text {. } \\
\text { Índice de condición: } 13,07 \text {. } \\
\mathrm{R}^{2} \text { (coeficiente de determinación ajustado): } 0,732 \text {. } \\
\mathrm{R}^{2} \text { (coeficiente de determinación): } 0,758 \text {. } \\
\mathrm{R} \text { (coeficiente de correlación): } 0,871 \text {. }\end{array}$} \\
\hline
\end{tabular}

AEVMP por homicidio entre los distintos estados del país, como puede apreciarse al ver sus coeficientes de regresión estandarizados y su significación estadística.

El modelo construido es estadísticamente significativo; no presenta autocorrelación, de acuerdo con los valores de la prueba de Durbin-Watson (alrededor de 2), ni alta colinealidad, de acuerdo con el índice de condición (inferior a 20).

\section{DISCUSIÓN}

Entre 1990 y 2016 se registraron en México 428.012 homicidios, de ellos 380.262 fueron de hombres $(89 \%) ; 153.420$ de las víctimas masculinas (es decir, $40 \%$ del total de homicidios masculinos) tenían entre 15 y 29 años, de los cuales 20.180 ocurrieron entre 2014 y $2016^{(14,15)}$. Estos números apenas dan una idea de la magnitud de la violencia homicida en el país y de la repercusión social, demográfica y sanitaria de estas vidas truncas a edades tempranas.

De acuerdo con la OPS ${ }^{(32)}$, alrededor de 2015, la tasa de homicidio masculino en México (30 por 100.000 habitantes según OPS, 36,2 en el trienio 2014-2016 según el presente estudio) era similar a la tasa promedio de la región (31,7 por 100.000 habitantes). Aunque inferior a las tasas de El Salvador, Colombia, Venezuela y Brasil, la tasa de México era 15 veces mayor que la de Canadá $(2,0)$, casi cuadruplicó a las de Chile $(7,6)$ y Cuba $(8,2)$, casi triplicó las de $\operatorname{EEUU}(9,6)$, Argentina $(11,6)$ y Nicaragua $(11,3)$, y fue notoriamente superior a las de Costa Rica $(18,8)$ y Uruguay $(13,3)$.

La OPS reconoce al homicidio juvenil como la principal causa de muerte entre jóvenes en Las Américas (33) y las tasas más elevadas en la región se ubican en los grupos etarios de 20 a 24 y 25 a 29 años. Una situación semejante se presenta con el homicidio juvenil masculino, pues si bien la tasa de homicidios de 15 a 24 años en México 
en el período 2008-2012 (25 por 100.000 habitantes) estaba por debajo de las observadas en Colombia y Brasil, era definitivamente mayor a la reportada por Argentina alrededor de $2008^{(4,5)}$.

En el trienio 2014-2016, la tasa de homicidio masculino en México, del grupo de 15 a 29 años, fue de 49,8 por 100.000 habitantes, la cifra más alta reportada en los últimos 25 años. Esto permite entender por qué los homicidios son actualmente $-y$ desde 2008la principal causa de muerte de los hombres jóvenes de dicho grupo etario ${ }^{(14)}$.

Ahora bien, las altas tasas de homicidio masculino juvenil reportadas en los últimos años contrastan con el marcado descenso observado en la tasa en los primeros 15 años del período analizado. Existen argumentos mencionados por diferentes autores ${ }^{(2,3,6,34)}$ que pueden ayudar a comprender tanto el descenso como el ascenso de la tasa a partir de mediados de la primera década del presente siglo.

En términos generales, la disminución observada parece estar relacionada con ciertas mejoras estructurales, como el crecimiento paulatino del producto interno bruto per cápita, una leve reducción de los niveles de pobreza y las bajas tasas de desempleo, aunque también con la moderación de la intensidad de los enfrentamientos entre cárteles del crimen organizado a partir del establecimiento de la llamada "federación" (alianza entre varios cárteles, bajo el liderazgo del cártel de Sinaloa) de fines de la década de 1990 hasta 2004-, entre otros aspectos ${ }^{(35)}$.

Sin embargo, la renovación de la violencia y el aumento de los homicidios, en especial el homicidio juvenil masculino, no responde solo al empeoramiento de estas condiciones estructurales -lo cual sin dudas ha sucedido, como es el caso del repunte del porcentaje de personas en condición de pobreza a partir de $2008^{(30)}$ o la reducción del producto interno bruto per cápita- sino también al efecto de factores coyunturales, como el empoderamiento de los grupos criminales dedicados al tráfico de drogas y otras actividades ilícitas, en contubernio con autoridades de diferentes niveles de gobierno ${ }^{(2,36)}$; la intensificación de la disputa que mantienen los cárteles del narcotráfico por el control de territorios, dadas las dificultades para acceder al mercado estadounidense de la droga ${ }^{(37)}$; la guerra contra el crimen organizado emprendida por el gobierno mexicano a partir de 2007 (con el despliegue del ejército en gran parte del país), los altos niveles de impunidad y corrupción existentes en los sistemas de seguridad y justicia del país ${ }^{(36,37,38)}$ y la cantidad cada vez mayor de armas de fuego en poder de civiles, las cuales ingresan mayoritariamente de manera ilegal por la frontera con EEUU ${ }^{(39)}$.

Asimismo, existen contextos que contribuyen específicamente al crecimiento de la violencia entre la población juvenil: por una parte, la falta de empleos -o la precarización del mismo, con salarios entre los más bajos del continente-; por otra, la carencia de plazas suficientes para estudiar en universidades públicas y la baja calidad educativa en los niveles previos, todo esto unido al hecho de que las generaciones actuales de adolescentes y jóvenes son las más numerosas en la historia del país, ha generado un notorio número de jóvenes entre 15 y 29 años que no tienen empleo ni están inscritos en educación o formación ${ }^{(40)}$; junto a la marcada desigualdad social existente en el país y a la degradación del espacio urbano, donde muchos jóvenes conviven cotidianamente con conductas altamente violentas ${ }^{(41)}$, son todos estos elementos que favorecen el incremento de la probabilidad de los jóvenes de estar expuestos a cometer o ser víctima de hechos delictivos, de integrarse a una pandiIla o ser reclutados por el crimen organizado como sicarios, informantes o distribuidores de drogas ${ }^{(42,43)}$.

A partir del aumento generalizado de la violencia en la última década, diversos autores han documentado la relación entre muertes violentas y esperanza de vida $y$, en especial, algunos de ellos han hecho énfasis en la importancia de los homicidios -mayormente los que ocurren en edades jóvenes- en la ralentización del incremento de la esperanza de vida en México, o incluso en su estancamiento o reducción en el caso de la esperanza de vida masculina ${ }^{(44)}$. Así, una comparación entre Brasil y México puso de 
relieve que los AEVMP por los homicidios entre 15 y 29 años aumentaron entre 20022004 y 2012-2014 -sobre todo en México, aunque la cifra en Brasil era aun considerablemente mayor- y representaban casi el 50\% del total de AEVMP en esas edades en Brasil y el $30 \%$ en México, por lo que tenían un impacto negativo en la esperanza de vida de ambas naciones ${ }^{(10)}$. Otro estudio corrobora la contribución negativa de los homicidios masculinos en el grupo de 15 a 29 al cambio de la esperanza de vida en México entre 2000 y $2013^{(12)}$ y un trabajo previo comparando Colombia y México, también evidencia cómo el grupo etario de 15 a 29 años es responsable del $70 \%$ de los AEVMP por homicidios en Colombia en 2011 $1^{(45)}$.

A tono con lo anterior, los hallazgos de la presente investigación revelan, en primer lugar, que en la última década los niveles de homicidio masculino en el grupo de 15 a 29 años aumentaron no solo en el país, sino en todas las entidades federativas (excepto Nayarit) -aún en aquellas donde tradicionalmente se han observado las tasas más bajas- y que este crecimiento fue muy marcado en la mitad de ellos, en los cuales los AEVMP por homicidios en este grupo etario en 2014-2016 al menos doblaron lo estimado para 2004-2006.

Por otra parte, se pone de manifiesto el papel que ha desempeñado el homicidio masculino juvenil en los cambios observados en la EVTM: en todas las entidades federativas en donde se redujo la EVTM se incrementaron sustancialmente los AEVMP por homicidio en el grupo de 15 a 29 años, y en especial en aquellos estados donde la EVTM se redujo más -Guerrero Colima, Sinaloa, Zacatecas, o que tienen una EVTM más baja, como Chihuahua- los aumentos en los AEVMP por homicidio en el grupo de 15 a 29 años fueron más acentuados.

El hecho de que en ocho estados el número de AEVMP por homicidio en el grupo de 15 a 29 años en el trienio 2014-2016 sea mayor a 0,5 (es decir, medio año de vida perdido) - cuando en 2004-2006 la cifra más alta la presentaba Chihuahua y era 0,42 - revela la trascendencia de la violencia homicida masculina juvenil para la esperanza de vida y la magnitud de su crecimiento en el último decenio, además del peso adquirido en el total de AEVMP en este grupo etario.

Así, el impacto del homicidio juvenil en la expectativa de vida masculina es claro: las altas tasas de homicidio en edades jóvenes llevan implícito que las cuantiosas víctimas que fallecen entre los 15 y 29 años no vivan los años que realmente podrían vivir si murieran de muerte natural, de acuerdo con los niveles de esperanza de vida existentes actualmente, a diferencia de lo que sucede con las muertes por enfermedades crónicas, que se concentran en edades más avanzadas.

Como se ha señalado previamente, en casi todas las entidades federativas se incrementaron los AEVMP a causa del homicidio masculino juvenil, pero en realidad los niveles son disímiles. En la práctica, los resultados sugieren que en aquellas entidades en las que el ejército destruye más hectáreas cultivadas de mariguana y opiáceas, en las que la población percibe una mayor inseguridad y donde el ejército confisca un mayor número de armas de fuego, la cifra de AEVMP por homicidio en el grupo de 15 a 29 años tiende a ser más elevada y viceversa.

Así, el análisis de las variaciones interestatales en el trienio 2014-2016 parece confirmar algunos de los argumentos expuestos con anterioridad, en especial la relevancia del crimen organizado (y sobre todo la presencia de los carteles del narcotráfico) junto a la alta disponibilidad de armas de fuego -incluso de grueso calibre- para explicar los altos valores que alcanza el indicador AEVMP por homicidio en el grupo 15 a 29 años en estados como Chihuahua, Guerrero, Sinaloa, Colima, Zacatecas o Tamaulipas. El carácter fronterizo de Chihuahua o Tamaulipas facilita la introducción ilegal de armas de fuego procedentes de EEUU y su uso indiscriminado por los grupos criminales asentados en estos estados o en entidades relativamente cercanas como Sinaloa y Zacatecas, grupos que se convierten con frecuencia en reclutadores de jóvenes para sus actividades ilícitas o incluso en ejemplos de cómo enriquecerse y obtener poder ${ }^{(2)}$.

$\mathrm{Si}$ bien entidades como Chihuahua, 
Guerrero, Sinaloa y Michoacán se mantuvieron entre los estados que registraron en ambos trienios los índices de homicidio juvenil más altos, el marcado crecimiento de los AEVMP por homicidio juvenil en Colima, Zacatecas, Nuevo León o Guanajuato parece reflejar una reconfiguración de la actividad criminal y un reacomodo de los cárteles del narcotráfico, como respuesta al proceso discursivo del gobierno que legitima la persecución del tráfico de drogas y el empleo de las fuerzas armadas para ello, lo que modifica también las formas de apropiación del territorio por parte de los grupos delincuenciales ${ }^{(46)}$.

A su vez, la percepción de inseguridad existente -sobre todo en los espacios públicos- es en gran medida reflejo del clima de terror e impunidad que reina en el país, con autoridades federales, estatales y municipales que no son capaces de garantizar la seguridad de sus ciudadanos, lo que ha propiciado una banalización de la violencia común que se ha incrementado drásticamente, al igual que los abusos policiales-y, por ende, un cambio en el comportamiento cotidiano de la población ${ }^{(2,28)}$. En Guerrero, Chihuahua, Michoacán, Zacatecas y Tamaulipas -todos estados con más de 0,5 AEVMP por homicidio juvenil- la percepción de inseguridad es claramente superior a la media nacional(28).

Por el contrario, casos como los estados de Yucatán, Aguascalientes o Tlaxcala, con un reducido número de AEVMP por homicidio juvenil, ponen de relieve que en aquellos sitios donde la presencia del crimen organizado y los cárteles del narcotráfico es menos notoria, y la sensación de inseguridad menor, el impacto del homicidio entre los hombres de 15 a 29 años en la esperanza de vida masculina es obviamente más reducido.

Aun cuando el presente estudio puede tener algunas limitaciones, como el posible subregistro de la mortalidad existente en las regiones de mayor marginación del país -el cual pudiera afectar la construcción de las tablas de mortalidad en algunos estados y por tanto su comparación- o los posibles errores en la recolección o clasificación de las defunciones como homicidios, no existen evidencias que permitan inferir que los resultados obtenidos para cada trienio y el análisis realizado a partir de estos puedan estar significativamente distorsionados, dado que la mejoría en los registros de defunciones ha sido generalizada en el país en años recientes ${ }^{(47)}$. En todo caso, esto significaría que el impacto del homicidio juvenil en la esperanza de vida podría estar subestimado. El propio hecho antes mencionado de la calificación satisfactoria por parte de la OPS de los registros de mortalidad en México ${ }^{(18)}$ avala la confianza en los hallazgos obtenidos.

Por otra parte, el empleo del supuesto de mortalidad nula -entre tres posibles opciones- para el cálculo de los AEVMP no altera el sentido de los resultados y es recomendado por la bibliografía ${ }^{(26)}$ en tanto facilita la interpretación de los resultados y explica en su totalidad los cambios en la EVTM.

A lo anterior habría que agregar la conocida "falacia ecológica", en tanto la asociación observada entre variables agregadas no necesariamente se replica a nivel individual. En este caso, sin embargo, el interés estribaba más en perfilar un contexto explicativo de las diferencias encontradas en el comportamiento del homicidio juvenil en las entidades federativas que en identificar atributos individuales asociados a ser víctima de homicidio entre los 15 y 29 años.

A pesar de las posibles limitaciones antes mencionadas, los resultados del estudio permiten afirmar con razonable certeza, por una parte, que la violencia homicida que afecta a los hombres entre los 15 y 29 años se ha incrementado marcadamente en la última década en México y, en la práctica, en todas las entidades federativas del país. Por otra, demuestran el impacto de los homicidios en edades jóvenes en la esperanza de vida masculina en México y en la mayoría de los estados. Es evidente que la esperanza de vida masculina podría crecer de manera palpable si los homicidios masculinos en las edades jóvenes se redujeran de forma sustancial.

Es obvio que el sistema de salud no puede hacer frente por sí solo a una cuestión de tanta envergadura como el homicidio juvenil. La salud pública -a diferencia del sistema judicial, orientado a apresar y castigar a 
quienes infringen la ley- debe colaborar de forma más activa en determinar la magnitud del problema, su alcance y características, así como en identificar aquellos factores que favorecen la violencia juvenil y qué se puede hacer para modificarlos. Desde una perspectiva poco usual, este estudio aporta elementos para entender la dimensión sociosanitaria del homicidio juvenil -en particular el que afecta a los hombres- y la necesidad de su abordaje interdisciplinario si se quiere tener una población joven con menos riesgo de morir prematuramente víctima de la violencia.

Así, los programas conducentes a disminuir la violencia juvenil $-y$, por ende, las tasas de homicidio en estas edades- deben considerarse en el contexto de una política integral que tome en cuenta las diversas dimensiones de la violencia ${ }^{(48)}$, pues si no se tiene una estrategia adecuada para satisfacer necesidades básicas y afrontar temas como la pobreza, la desigualdad social y el desempleo, junto a la necesidad de reducir los niveles de impunidad y corrupción existentes, de implementar acciones tendientes a reducir el número de armas de fuego ilegales que circulan en el país y de reevaluar las políticas existentes para reducir el tráfico de drogas ilegales y limitar su producción, distribución y consumo entre los jóvenes, difícilmente se reducirá el número de muertes juveniles masculinas por esta causa ${ }^{(49)}$. Asimismo, es necesario el cuestionamiento de los valores predominantes en la sociedad sobre la masculinidad, ideas que se transfieren de generación en generación a través del proceso de socialización, y que llevan implícito un modo de vida que involucra a los hombres con mayor frecuencia que a las mujeres en conductas violentas, riesgosas y extremas que los exponen a una mayor probabilidad de muerte por esta causa ${ }^{(50)}$.

\section{REFERENCIAS BIBLIOGRÁFICAS}

1. Programa de las Naciones Unidas para el Desarrollo. Informe Regional de Desarrollo Humano 2013-2014; Seguridad ciudadana con rostro humano: Diagnóstico y propuestas para América Latina [Internet]. Panamá: PNUD; 2013 [citado 20 nov 2017]. Disponible en: https://tinyurl.com/ h3z594b.

2. Bataillon G. Narcotráfico y corrupción: las formas de la violencia en México en el siglo XXI. Nueva Sociedad. 2015;(255):54-68.

3. González-Pérez GJ, Vega-López MG, Muñoz de la Torre A. Juventud, homicidio y esperanza de vida en México. En: Gutiérrez F, (coord.). Conducta violenta: Impacto biopsicosocial. Guadalajara: Editorial Universitaria; 2016. p. 41-62.

4. Alvarado A. Mortalidad juvenil en México. En: Alvarado A, Concha-Eastman A, Spinelli H, Peres MFT. Vidas Truncadas: el exceso de homicidios en la juventud de América Latina, 1990-2010. México DF: El Colegio de México; 2015. p. 171-212.

5. Alvarado A. La violencia juvenil en América Latina. Estudios Sociológicos. 2013;31(91):229-258.
6. González-Pérez GJ, Vega-López MG, CabreraPivaral CE, Vega-López A, Muñoz de la Torre A. Mortalidad por homicidios en México: tendencias, variaciones socio-geográficas y factores asociados. Ciência \& Saúde Coletiva. 2012;17(11):3195-3208.

7. Short Jr J. Poverty, ethnicity and violent crime. New York: Routledge; 1997.

8. Souza ER. Homicídios: metáfora de uma nação autofágica. [Teses de Doutorado]. Rio de Janeiro: Escola Nacional de Saúde Pública; 1995.

9. Haagsma JA, Graetz N, Bolliger I, Naghavi M, Higashi $\mathrm{H}$, Mullany EC, et al. The global burden of injury: incidence, mortality, disability-adjusted life years and time trends from the Global Burden of Disease study 2013. Injury Prevention. 2016;22(1):3189. doi: 10.1136/injuryprev-2015-041616.

10. González-Pérez GJ, Vega-Lopez MG, Ramos de Souza E, Wernersbach Pinto L. Mortalidad por violencias y su impacto en la esperanza de vida: una comparación entre México y Brasil. Ciência \& Saúde Coletiva. 2017;22(9):2797-2809.

11. Aburto JM, Beltrán-Sánchez H, García-Guerrero VM, Canudas-Romo V. Homicides in Mexico reversed life expectancy gains for men and slowed them 
for women, 2000-10. Health Affairs. 2016;35(1):8895.

12. Dávila Cervantes CA, Pardo Montaño AM. Análisis de la tendencia e impacto de la mortalidad por causas externas: México, 2000-2013. Salud Colectiva. 2016;12(2):251-264. doi: 10.18294/ sc. 2016.743 .

13. González-Pérez GJ, Vega-López MG, CabreraPivaral CE. Impacto de la violencia homicida en la esperanza de vida masculina de México. Revista Panamericana de Salud Pública. 2012;32(5):335-342.

14. Base de datos de defunciones 1979-2015 [Internet]. México DF: Dirección General de Información en Salud; 2015 [citado 10 oct 2017]. Disponible en: https://tinyurl.com/y8fkvjkg.

15. Mortalidad; Conjunto de datos: Defunciones por homicidios 2016 [Internet]. Aguascalientes: Instituto Nacional de Estadística y Geografía; 2016 [citado 19 nov 2017]. Disponible en: https:// tinyurl.com/y9452acn.

16. Consejo Nacional de Población. Estimaciones (1990-2010). En: Proyecciones de la población 2010-2050 [Internet]. México DF: CONAPO; 2014 [citado 13 oct 2017]. Disponible en: https:// tinyurl.com/ycchkabf.

17. Organización Mundial de la Salud. Clasificación estadística internacional de enfermedades y problemas relacionados con la salud [Internet]. Vol. 1. Washington: OPS; 1995 [citado 13 oct 2017]. Disponible en: https://tinyurl.com/ycjmbv4u.

18. Organización Panamericana de la Salud. Situación de salud en las Américas: Indicadores básicos 2015. Washington: OPS; 2015.

19. Särndal C, Swensson B, Wretman J. Basic ideas in estimation from probability samples. En: Särndal C, Swensson B, Wretman J, (eds.). Model Assisted Survey Sampling. New York: Springer; 1997. p. 24-58.

20. Comisión Económica para América Latina. La juventud en Iberoamérica: Tendencias y urgencias [Internet]. Santiago de Chile: CEPAL; 2004. [citado 13 oct 2017]. Disponible en: https://tinyurl.com/ y9wr9h3w.

21. Instituto Nacional de Estadística y Geografía. Estadísticas a propósito del día internacional de la juventud (15 a 29 años). Aguascalientes: INEGI; 2016.

22. Xunta de Galicia. Epidat [Internet]. Versión 4.2. La Coruña: Xunta de Galicia, OPS; 2006 [citado 13 oct 2017]. Disponible en: https://tinyurl.com/ yb25kx8j.

23. Arriaga EE. Los años de vida perdidos: su utilización para medir el nivel y el cambio de la mortalidad. Notas de Población. 1996;24(63):7-38.

24. Arriaga EE. Measuring and explaining the change in life expectancies. Demography. 1984; 21(1):83-96.

25. Bocco M. La relación entre los años de vida perdidos y la esperanza de vida: aplicaciones para el análisis de la mortalidad. Notas de Población. 1996;24(63):39-60.

26. Bradshaw J, Edwards H, Lawton D, Staden F, Weale J, Weekes A. Area variations in infant mortality 1975-1977. Journal of Epidemiology and Community Health. 1982;36:11-16.

27. Murnan J, Dake JA, Price JH. Association of selected risk factors with variation in child and adolescent firearm mortality by state. The Journal of School Health. 2004;74(8):335-340.

28. Instituto Nacional de Estadística y Geografía. Encuesta nacional de victimización y percepción sobre seguridad pública (ENVIPE) 2015 [Internet]. Aguascalientes: INEGI; c2015 [citado 13 oct 2017]. Disponible en: https://tinyurl.com/ ycddtj92.

29. Instituto Nacional de Estadística y Geografía. Anuario estadístico y geográfico por entidad federativa 2016 [Internet]. Aguascalientes: INEGI; 2016 [citado 13 oct 2017]. Disponible en: https:// tinyurl.com/y82xgnc4.

30. Consejo Nacional de Evaluación de la Política de Desarrollo Social. Anexo estadístico de pobreza en México [Internet]. México DF: CONEVAL; c2016 [citado 17 nov 2017]. Disponible en: https://tinyurl.com/ydhjzodp.

31. Instituto Nacional de Estadística y Geografía. Anuario estadístico y geográfico por entidad federativa 2013 [Internet]. Aguascalientes: INEGI; 2014 [citado 17 nov 2017]. Disponible en: https:// tinyurl.com/ycf6zcao.

32. Etienne CF, (dir.). Situación de salud en las Américas: Indicadores básicos 2017 [Internet]. Washington DC: OPS; 2017 [citado 17 nov 2017]. Disponible en: https://tinyurl.com/y7nv2u7t.

33. Organización Panamericana de la Salud. Homicidio en jóvenes en las Américas [Internet]. Washington DC: OPS; 2017 [citado 27 nov 2017] Disponible en: https://tinyurl.com/y7b8c7h3.

34. Escalante Gonzalbo F. Homicidios 2008-2009: 
La muerte tiene permiso. Nexos. 2011;397:36-49.

35. Montalvo TL. Una guerra por el dominio del país: la rivalidad entre organizaciones criminales. Narcodata [Internet]; c2015 [citado 18 ago 2016]. Disponible en: https://tinyurl.com/yb7mreoo.

36. González Veiguela L. El crecimiento del "narco" no es posible sin la complicidad de las autoridades. Esglobal [Internet]; 26 ene 2010 [citado 10 nov 2015]. Disponible en: https://tinyurl.com/ y8rfwros.

37. González Veiguela L. Los cárteles más fuertes de México. Esglobal [Internet]; 28 jun 2018 [citado 10 nov 2015]. Disponible en: https://tinyurl.com/ y832nr2x.

38. Astorga L. Seguridad, traficantes y militares: El poder y la sombra; Tiempo de Memoria. México DF: Tusquets; 2007.

39. Goodman C, Marizco M. U.S. Firearms trafficking to Mexico: new data and insights illuminate key trends and challenges 2010 [Internet]. Washington DC: Woodrow Wilson Center; 2010 [citado 12 sep 2016]. Disponible en: https://tinyurl. com/yaono5ya.

40. Organización para la Cooperación y el Desarrollo Económicos. Panorama de la Educación 2014: Indicadores de la OCDE. Organización para la Cooperación y el Desarrollo Económicos [Internet]; c2014 [citado 15 oct 2017]. Disponible en: https://tinyurl.com/yc2qathy.

41. Orraca Corona M. Desigualdades, juventudes y violencia. En: Herrera Lasso Mijares L, (coord.). Fenomenología de la violencia: Una perspectiva desde México. México DF: Siglo XXI Editores; 2017.
42. Thornberry T, Krohn M, Lizotte A, Smith C, Tobin K. Gangs and delinquency in developmental perspective. Cambridge: Cambridge University Press; 2003.

43. Villaveces A, Cummings P, Espitia VE, Koepsell TD, McKnight B, Kellermann AL. Effect of a ban on carrying firearms on homicide rates in $2 \mathrm{Co}-$ lombian cities. JAMA. 2000;283(9):1205-1209.

44. Pérez Gómez LE. Esperanza de vida en México: De cómo los indicadores pueden señalar catástrofes. Perseo. 2013;(10). Disponible en: https://tinyurl.com/ybjfx3wh.

45. Dávila-Cervantes CA, Pardo-Montaño AM. Magnitud y tendencia de la mortalidad por homicidios en Colombia y México, 2000-2011. Revista Panamericana de Salud Pública. 2014;36(1):10-16.

46. Fuerte-Celis MP, Pérez-Lujan E. ¡La violencia cambia!: Securitización y configuración territorial del crimen organizado en México, 2007-2011. Journal of Latin American Geography. 2017;16 (3):59-81.

47. Lozano-Ascencio R. ¿Es posible seguir mejorando los registros de las defunciones en México? Gaceta Médica de México. 2008;144(6):525-533.

48. Zunino MG, Souza ER. La mortalidad por armas de fuego en Argentina entre 1990 y 2008. Cadernos de Saúde Pública. 2012;28(4):665-677.

49. Gamlin J. Violence and homicide in Mexico: A global health issue. The Lancet. 2015;385:605-606.

50. Souza ER. Masculinidade e violência no Brasil: contribuições para a reflexão no campo da saúde. Ciência \& Saúde Coletiva. 2005;10(1):59-70. doi: 10.1590/S1413-81232005000100012.

Recibido: 23 de enero de 2018 | Versión final: 8 de noviembre de 2018 | Aprobado: 12 de diciembre de 2018

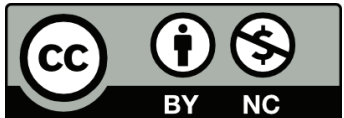

Esta obra está bajo una licencia de Creative Commons Reconocimiento-NoComercial 4.0 Internacional. Reconocimiento - Permite copiar, distribuir y comunicar públicamente la obra. A cambio, se debe reconocer y citar al autor original. No Comercial — Esta obra no puede ser utilizada con finalidades comerciales, a menos que se obtenga el permiso. 